\title{
A compact simple microstrip lowpass filter based on elliptical analyzed resonator
}

https://doi.org/10.1515/freq-2021-0157

Received June 30, 2021; accepted November 10, 2021;

published online November 29, 2021

\begin{abstract}
In this article, a compact microstrip lowpass filter (LPF) using elliptical shaped resonators with ultra-wide stopband is rendered. In this respect, LC equal structures of the elliptical shaped resonators are calculated based on the formula of circumference. In addition, to calculate transmission zeros of the presented elliptical shaped resonator, the LC equal structure and its output to input ratio are employed. The proposed LPF has a $-3 \mathrm{~dB}$ cut-off frequency at $1.50 \mathrm{GHz}$ and the stopband bandwidth of the designed filter is about $13 f \mathrm{f}$, which refers to its ultra-wide stopband. The occupied circuit size of the presented filter is $0.151 \lambda_{g} \times 0.044 \lambda_{g}$ $\left(\lambda_{g}\right.$ is the guided wavelength at $\left.1.50 \mathrm{GHz}\right)$. The designed filter is fabricated on RT/Duroid 5880 substrate. The results of the fabricated and designed filter have clearly demonstrated that not only has the proposed LPF shown a suitable agreement between measured and simulated $S$-parameters, but also an appropriate stopband bandwidth.
\end{abstract}

Keywords: elliptical shaped resonator; LC equal structure; lowpass filter (LPF); output to input ratio; transmission zero; ultra-wide stopband.

\section{Introduction}

Compact microstrip LPFs with ultra-wide stopband are extremely desirable for wireless and mobile communication

*Corresponding author: Saeedeh Lotfi, Department of Materials and Technology, University of West Bohemia in Pilsen, Plzen, Czech Republic, E-mail: lotfi@fel.zcu.cz.

https://orcid.org/0000-0003-4110-7202

Mohsen Hayati, Department of Electrical Engineering, Razi University, Kermanshah, Iran

Hesam Siahkamari and Richard Linhart, Department of Electronics and Information Technology, University of West Bohemia in Pilsen, Plzen, Czech Republic

Tomas Blecha, Department of Materials and Technology, University of West Bohemia in Pilsen, Plzen, Czech Republic

Mohammad (Behdad) Jamshidi, Research and Innovation Center for Electrical Engineering (RICE), University of West Bohemia in Pilsen, Plzen, Czech Republic systems to suppress unwanted high frequency harmonics. These systems need the LPF circuits with high performance. Therefore, LPFs with good frequency features are very noticeable in many communication systems [1, 2]. Several studies have been done to implement and analyze LPFs and microwave circuits with high performance [3-31]. One method, which is used in LPFs implementation, is Defected Ground Structure (DGS) to obtain high stopband suppression and size reduction [3, 4]. Another method is using stubloaded resonators such as a compact LPF with a wide stopband, which is presented in [5]. Using coupled rhombic stubs, a compact and highly selective LPF with a wide stopband is presented in [6]. Authors in [7] applied hexangular shaped resonators for designing a LPF with high performance. Another LPF with compact size and sharp rolloff using modified Complementary Split-Ring Resonator (CSRR) was presented in [8]. A compact harmonic suppressed microstrip structure is presented in [9], which consists of stepped impedance lines and symmetric modified semicircular patches. The dual-plane structure is another method that is used to design compact LPFs as mentioned in [10]. Another type of LPF is presented in [11], which is called multi stub LPF, in this structure the open stubs are used instead of impedance lines. A compact microstrip LPF with ultra-wide stopband is designed in [12], which uses an analyzed triangular shaped resonator in its design. Using a lattice-shaped resonator, a compact LPF with flat group delay with a wide rejection band and sharp roll-off is presented in [13]. Another LPF with sharp roll-off and ultra-wide stopband using circular sectors and cross-coupled structures is designed in [14]. A very applicable procedure for designing a lowpass filter is presented in [15], which some polygonal structures are embedded in its design and its analysis has been done based on transfer function evaluation. In [16], using LPF design, a dual-wideband bandpass filter with a wide upper stopband is presented, which uses a short stepped-impedance stubloaded resonator in its LPF design. Another LPF with compact and ultra-wide stopband is designed in [17], which uses a narrow width microstrip line with high impedance and three multimode resonators in its structure. Using fractal structures and hairpin units are other methods to design the LPFs, such as [18-20]. Authors in [21] applied non-uniform radial stubs resonators to design another compact microstrip 
LPF with a wide stopband. A new method to enhance the LPFs performances is using neural network analysis, in [22], another LPF is designed and fabricated using multiple open stubs and stepped impedance resonators, which its features have been improved utilizing the mentioned method. Another lowpass filter with a sharp rejection band using coupled T-shaped, elliptical and radial resonators is presented in [23]. Using four high-low impedance patches and four radial stub structures, a lowpass filter with efficient performances is designed in [24], which has a high relative stopband bandwidth. Based on inductor-capacitor equivalent circuit analysis and using some series resonators with meandered lines, another LPF is designed in [25]. Another method to design and analyze the filters and other microstrip devices is using even and odd mode technique, [26, 27]. Recently, LPFs are used to develop other microwave devices for example [28-31].

In this paper, a microstrip LPF with considerable features, such as ultra-wide stopband, compact size and simple design with a return loss better than $29 \mathrm{~dB}$ is presented. The proposed LPF with $-3 \mathrm{~dB}$ cut-off frequency at $1.50 \mathrm{GHz}$ is designed using elliptical resonators. In addition to the good features of the suggested design, a new formula is presented to calculate the LC equivalent structure of the elliptical structures based on its geometric circumference, which is a new technique to calculate the LC elements.

\section{Filter design}

The filter design consists of creating some elliptical resonators and computing their output to input ratios (transfer functions), to analyze their behavior. The elliptical resonators are selected due to their suitable frequency responses, and by applying these types of resonators in different sizes a filter with sharp transition band and wide stopband can be designed. Also, based on its circumference a new formula for calculating LC equivalent circuit can be computed and rendered.

\subsection{First elliptical resonator (FER)}

The topology and the LC equivalent structure of the first elliptical resonator are shown in Figure 1(a) and (b), respectively. Also, the Electro Magnetic (EM) and LC frequency responses of the FER are illustrated in Figure 1(c), up to $10 \mathrm{GHz}$. The first transmission zero of this structure occurs at $9.30 \mathrm{GHz}$ with an attenuation level better than $48 \mathrm{~dB}$, which is suitable to design a wide stopband LPFs.
Dimensions of this resonator are (All in millimeters): $L_{1}=5.7$, $L_{2}=0.51, W_{1}=8.56, W_{2}=2, W_{3}=0.1$.

The values of capacitances $\left(C_{x}\right)$ and inductances $\left(L_{x}\right)$ are computed using open ends and high-low impedance lossless lines formulas, [1]. Equations (1) and (2) express these formulas, where $Z_{s}$ is the characteristic impedance of the lines and $\lambda_{g}$ in Eqs. (1) and (2) is calculated by the width of lines [1] (Note: $\lambda_{g}$ is the calculated guided wavelength at $1.50 \mathrm{GHz})$.

$$
\begin{aligned}
& L_{x}=\frac{1}{\omega} \times Z_{s} \times \sin \left(\frac{2 \pi}{\lambda_{g}} L\right) \\
& C_{x}=\frac{1}{\omega} \times \frac{1}{Z_{s}} \times \tan \left(\frac{\pi}{\lambda_{g}} L\right)
\end{aligned}
$$

To achieve the LC equivalent structure of the proposed elliptical resonators, new computed formulas (Eqs. (3) and (4)) are obtained as:

$$
W_{\text {new }}=\left\{\begin{array}{cc}
\frac{1}{4}\left(\pi \sqrt{\frac{W^{2}+L^{2}}{2}}\right)+\frac{2 L}{W}\left(\frac{W}{L}-1.12\right) & \text { for }:\left(\frac{W}{L}>1.12\right) \\
\frac{1}{4}\left(\pi \sqrt{\frac{W^{2}+L^{2}}{2}}\right)+\frac{3 L}{W}\left(\frac{W}{L}-1.12\right) & \text { for }:\left(\frac{W}{L}<1.12\right)
\end{array}\right\}
$$

Equation (3) is obtained based on the geometric circumference of the elliptical shaped structure. The elliptical resonator is formed using half of an elliptical shaped patch. The $W_{\text {new }}$ in Eq. (3) is approximately equal to $1 / 2$ of a semi circumference of an elliptical shaped patch. Therefore, the circumference of an elliptical shaped cell have to be multiplied by $1 / 4$, but the $1 / 4$ of the elliptical circumference has a small error in comparison with EM simulation. To decrease this error, a new formula is computed, based on linearization of error. So, if $W / L$ becomes equal to 1.12, the error will be equal to approximately zero. Therefore, $3 L / W$ and $2 L / W$ in the first and second lines of Eq. (3) are calculated for decreasing the error. The length $(L)$ and width $(W)$ in Eqs. (1) and (2) are replaced with $L_{\text {new }}$ and $W_{\text {new }}$ which are given in Eqs. (3) and (4) for achieving the values of the LC equivalent structure of the elliptical resonators. Then, using open ends formulas [1], inductances and capacitances are computed. Calculated values for the LC equivalent structure of the FER are as follows. These amounts are shown by $C_{f i}(\mathrm{pF})$ and $L_{f i}(\mathrm{nH})$, parameters (Note: the " $f$ " index is related to FER design and $i=1,2, \ldots)$.

$$
\begin{aligned}
C_{f 1} & =0.33, C_{f 2}=0.97, C_{f 3}=0.58, L_{f 1}=L_{f 2}=7.09, L_{f 3} \\
& =0.08, L_{f 4}=0.39 .
\end{aligned}
$$




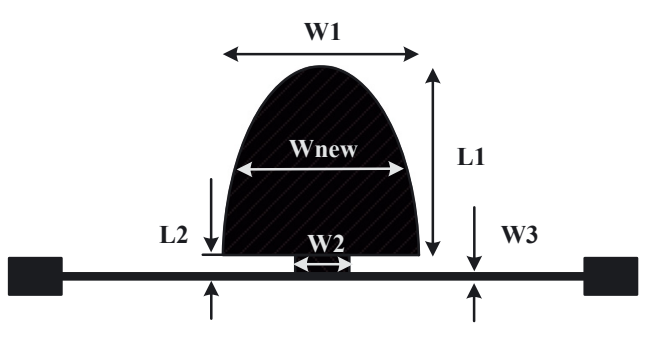

(a)

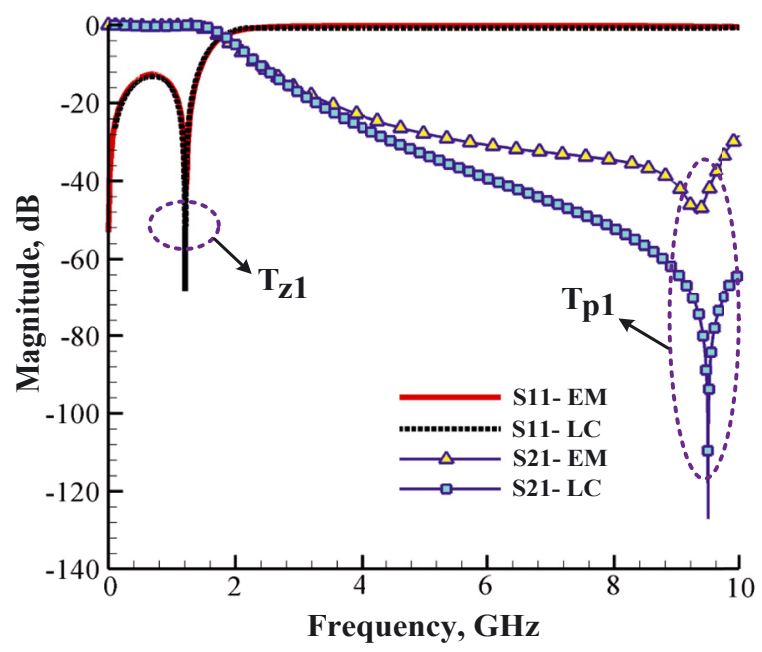

(c)

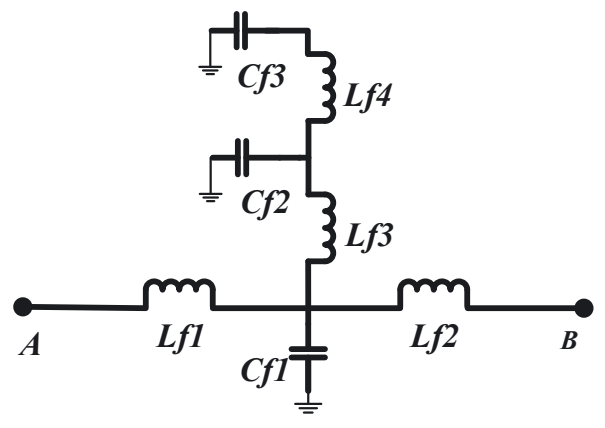

(b)

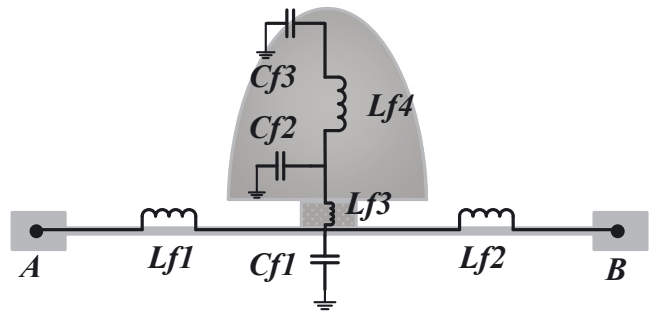

(d)

Figure 1: First elliptical resonator, (a) layout configuration, (b) LC circuit, (c) EM and LC simulation, (d) LC equivalent elements.

$L_{f 1}, L_{f 2}, L_{f 3}$ and $L_{f 4}$ (Eq. (1)) and $C_{f 1}$ (Eq. (2)) are denoted to the inductances and capacitance of high impedance lossless lines. $C_{f 2}$ is the summation of the capacitances of the resonator and high impedance lossless line (Eqs. (2)-(4)). $C_{f 3}$ denotes the capacitances of the resonator and its open-end effect (formulas of gap [1], Eqs. (3) and (4)). $L_{f 3}$ (Eqs. (3) and (4)) refers to the inductance of the investigated resonator. These LC equivalent elements are assigned and shown in Figure 1(d).

The output to input ratio of the investigated elliptical resonator is calculated in Eq. (5). The locations of transmission zeros can be determined by the output to input ratio. The designed structure can generate a deep transmission zero at $9.30 \mathrm{GHz}$, which is calculated in Eq. (6). It is shown in Figure 1(c) by $T_{z 1}$.

For example, $T_{z 1}$ can be changed by $L_{f 3}$ and $C_{f 2}$ based on Eq. (6), where other inductances and capacitances values are fixed. If $C_{f 2}$ is assumed as $0.94,0.97$ and $1.13 \mathrm{pF}$, and $L_{f 3}$ is assumed as $0.35,0.38$ and $0.42 \mathrm{nH}$, then $T_{z 1}$ will be 10.55 , 9.30 and $8.39 \mathrm{GHz}$, respectively. These calculated parameters are summarized in Table 1. For having mentioned transmission zeros, $L_{1}$ can be calculated about 5, 5.7 and $6.4 \mathrm{~mm}$, respectively, based on Eqs. (1) and (2).

$$
\frac{v_{o}}{v_{i}}=\frac{50 a}{\left(50+L_{f 1} S\right)\left(50+2 a+L_{f 1} S\right)}
$$

where: $(S=j \omega)$

$$
\begin{aligned}
& a=\frac{\left(1+\left(C_{f 2} L_{f 2}+C_{f 3}\left(L_{f 2}+L_{f 3}\right)\right) S^{2}+C_{f 2} C_{f 3} L_{f 2} L_{f f} S^{4}\right)}{\left(S\left(C_{f 1}+C_{f 2}+C_{f 3}+\left(C_{f 1}\left(C_{f 2}+C_{f 3}\right) L_{f 2}+\left(C_{f 1}+C_{f 2}\right) C_{f 3} S_{f 3}\right) C_{f 1} C_{f 2} C_{f 3} L_{f 2} L_{f 3} S^{4}\right)\right)} \\
& T_{z 1}=\frac{\sqrt{\frac{1}{C_{f 2} L_{f 2}}+\frac{1}{C_{f 2} L_{f 3}}+\frac{1}{C_{f 3} L_{f 3}}-\frac{\sqrt{-4 C_{f 2} C_{f 3} L_{f 2} L_{f 3}+\left(C_{f f} L_{f 2}+C_{f 3} L_{f 2}+C_{f 3} L_{f 3}\right)^{2}}}{C_{f 2} C_{f 3} f_{f 2} L_{f 3}}}}{2 \pi \sqrt{2}}
\end{aligned}
$$


Table 1: Different calculated amounts for $T_{\text {Z1-FER }}$.

\begin{tabular}{|c|c|c|c|c|c|}
\hline \multicolumn{2}{|c|}{$T_{Z 1 \text {-FER }}=10.55 \mathrm{GHz}$} & \multicolumn{2}{|c|}{$T_{Z 1-\mathrm{FER}}=9.30 \mathrm{GHz}$} & \multicolumn{2}{|c|}{$T_{Z 1-\mathrm{FER}}=8.39 \mathrm{GHz}$} \\
\hline$C_{f 2}$ & $0.94 \mathrm{pF}$ & $C_{f 2}$ & $0.97 \mathrm{pF}$ & $C_{f 2}$ & $1.13 \mathrm{pF}$ \\
\hline$L_{f 3}$ & $0.35 \mathrm{nH}$ & $L_{f 3}$ & $0.38 \mathrm{nH}$ & $L_{f 3}$ & $0.42 \mathrm{nH}$ \\
\hline
\end{tabular}

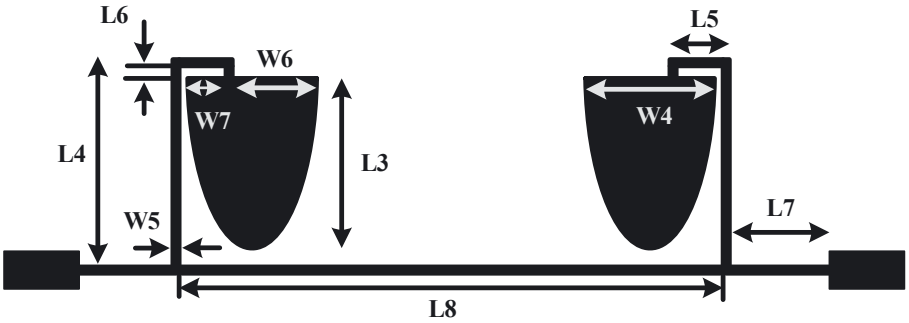

(a)

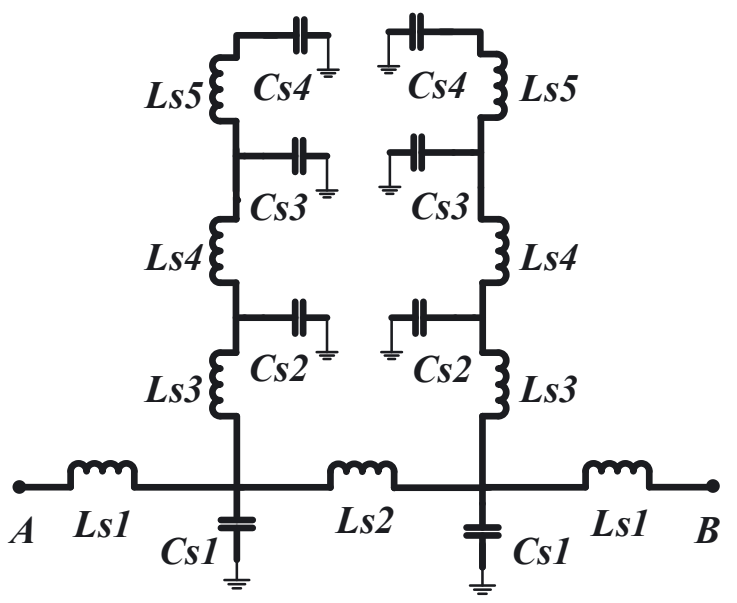

(b)

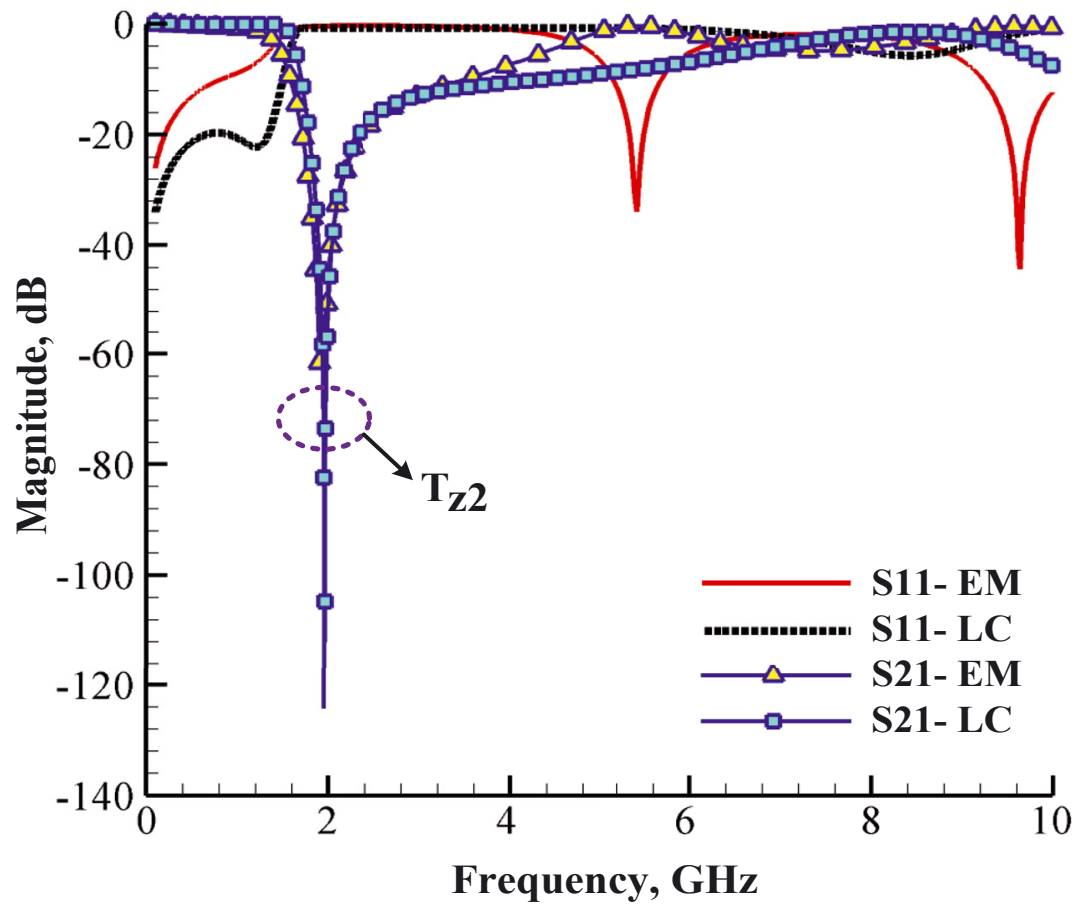

(c)
Figure 2: Second elliptical resonator (a) layout configuration, (b) LC equal structure, (c) EM and LC simulation. 


\subsection{Second elliptical resonator (SER)}

As discussed, the first transmission zero and pole of the FER are occur at $9.30 \mathrm{GHz}$ and $1.21 \mathrm{GHz}$, respectively, but this transmission zero and transmission pole are not near to each other, so the transition band is not sharp in this configuration. To improve the sharpness of the transition band, the SER is designed as seen in Figure 2(a). The LC equivalent structure of the SER is shown in Figure 2(b). The results of the EM and LC simulations are shown in Figure 2(c), up to $10 \mathrm{GHz}$.

The configuration of the SER generates a transmission zero at $1.97 \mathrm{GHz}$ with the attenuation level better than $48 \mathrm{~dB}$. The structure has a transmission pole at $1.08 \mathrm{GHz}$, too. So the transmission pole and zero are happen approximately near to each other; therefore, these double and smaller elliptical shaped structures helps to move the first LPF's transmission zero to the lower frequencies and leads to an increase in sharpness of the final LPF's transition band.

SER's dimensions are (All in millimeters): $L_{3}=5.45$, $L_{4}=6.4, L_{5}=2, L_{6}=0.19, L_{7}=2.54, L_{8}=16.7, W_{4}=4.17$, $W_{5}=0.1, W_{6}=2.44, W_{7}=1.63$. The LC equal values of the SER are shown by $C_{s i}(\mathrm{pF})$ and $L_{s i}(\mathrm{nH})$, parameters $(i=1,2,3 \ldots)$;

$$
\begin{aligned}
& L_{s 1}=1.7, L_{s 2}=7.11, L_{s 3}=4.23, L_{s 4}=1.39, L_{s 5}=0.52, \\
& C_{s 1}=0.27, C_{s 2}=0.11, C_{s 3}=0.45, C_{s 4}=0.60 .
\end{aligned}
$$

The output to input ratio of the LC equivalent structure for the SER, as shown in Figure 2(b), is calculated in Eq. (7). Using this ratio leads to determine the transmission zero locations (Note: $r=50 \Omega$ ).

$$
\frac{v_{o}}{v_{i}}=\frac{a^{2} r}{\left(a+r+L_{s 1} S\right)\left(2 a r+2 a L_{s 1} S+a L_{s 2} S+L_{s 2} r S+L_{s 1} L_{s 2} S^{2}\right)}
$$

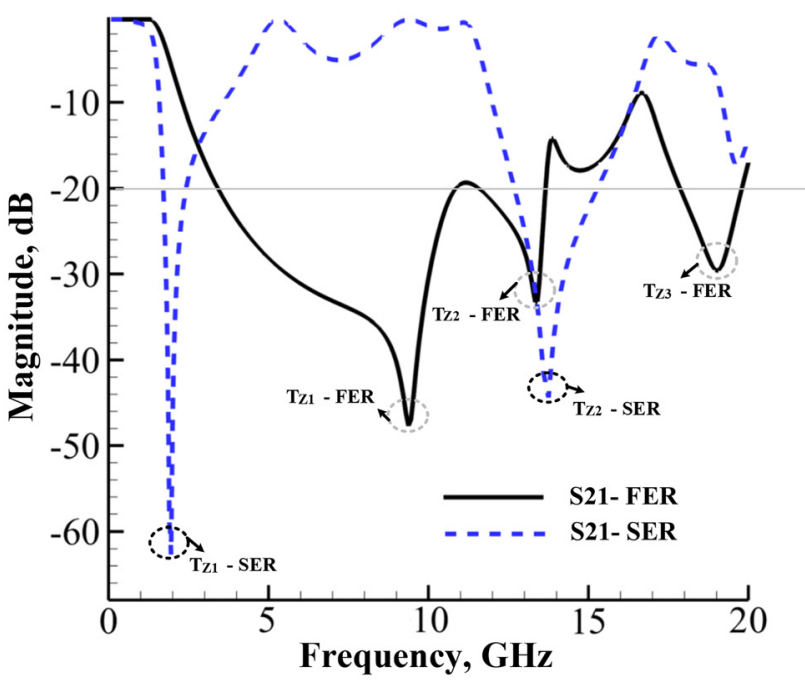

(a)

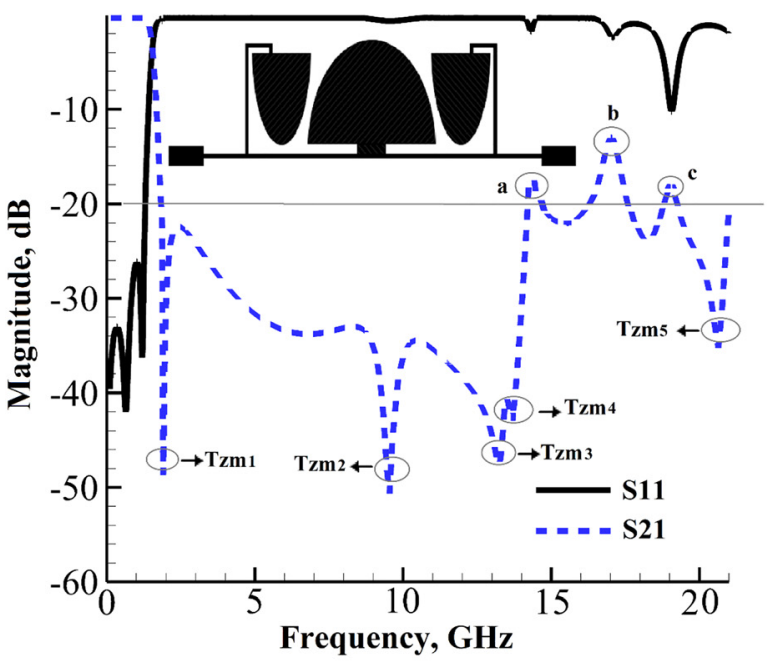

(b)

Figure 3: Frequency responses, (a) first and second elliptical resonators, (b) layout structure and frequency response of prototype LPF.

where:

$$
\begin{aligned}
a=\frac{\left(1+S^{2}\left(C_{s 4}\left(L_{s 3}+L_{s 4}+L_{s 5}\right)+C_{s 3}\left(L_{s 3}+L_{s 4}\right)\left(1+C_{s 4} L_{s 5} S^{2}\right)+C_{s 2} L_{s 3}\left(1+\left(C_{s 3} L_{s 4}+C_{s 4}\left(L_{s 4}+L_{s 5}\right)\right) S^{2}+C_{s 3} C_{s 4} L_{s 4} L_{s 5} S^{4}\right)\right)\right)}{\left(S \left(C_{s 1}+C_{s 2}+C_{s 3}+C_{s 4}+\left(C_{s 2}\left(C_{s 3}+C_{s 4}\right) L_{s 4}+\left(C_{s 2}+C_{s 3}\right) C_{s 4} L_{s 5}+C_{s 1}\left(C_{s 2} L_{s 3}+\left(C_{s 3}+C_{s 4}\right)\left(L_{s 3}+L_{s 4}\right)+C_{s 4} L_{s 5}\right)\right) S^{2}\right.\right.} \\
\\
\left.\left.\quad\left(C_{s 1} C_{s 2}\left(C_{s 3}+C_{s 4}\right) L_{s 3} L_{s 4}+C_{s 4}\left(C_{s 1}\left(C_{s 2}+C_{s 3}\right) L_{s 3}+\left(C_{s 1}+C_{s 2}\right) C_{s 3} L_{s 4}\right) L_{s 5}\right) S^{4}+C_{s 1} C_{s 2} C_{s 3} C_{s 4} L_{s 3} L_{s 4} L_{s 5} S^{6}\right)\right)
\end{aligned}
$$

\section{Prototype lowpass filter}

The simulation results of the FER and SER are shown in Figure 3(a), up to $20 \mathrm{GHz}$. As seen, the FER generates three transmission zeros and the SER can create two transmission zeros, too. In order to design a prototype LPF with a sharp transition band and wide stopband bandwidth, both first and second elliptical resonators are combined and a prototype LPF with five main transmission zeros plus some coupling effects is formed. This designed structure, and its simulation results are shown in Figure 3(b), while the transmission zeros are assigned by $T_{z m i}(i=1,2, \ldots, 5)$. As seen in Figure 3(b), this configuration has not a good suppression level in the stopband. 


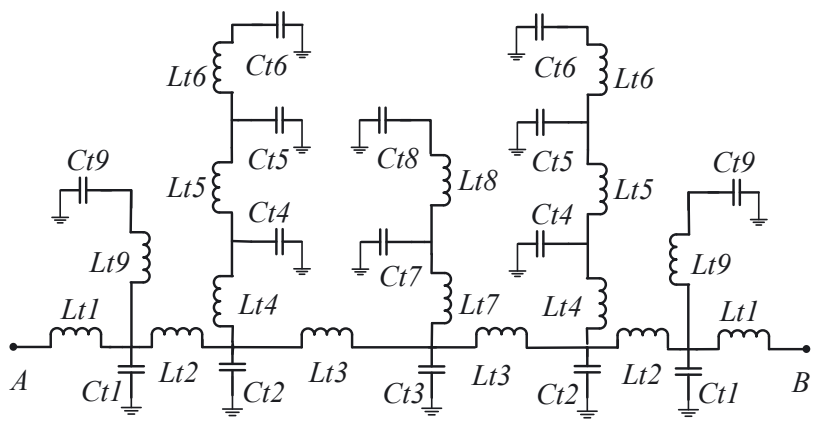

(a)

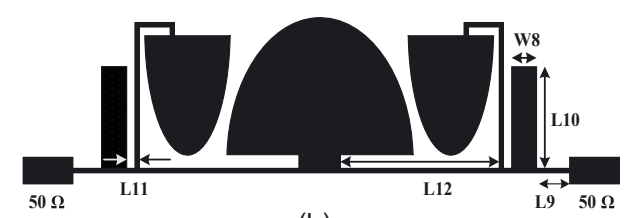

(b)

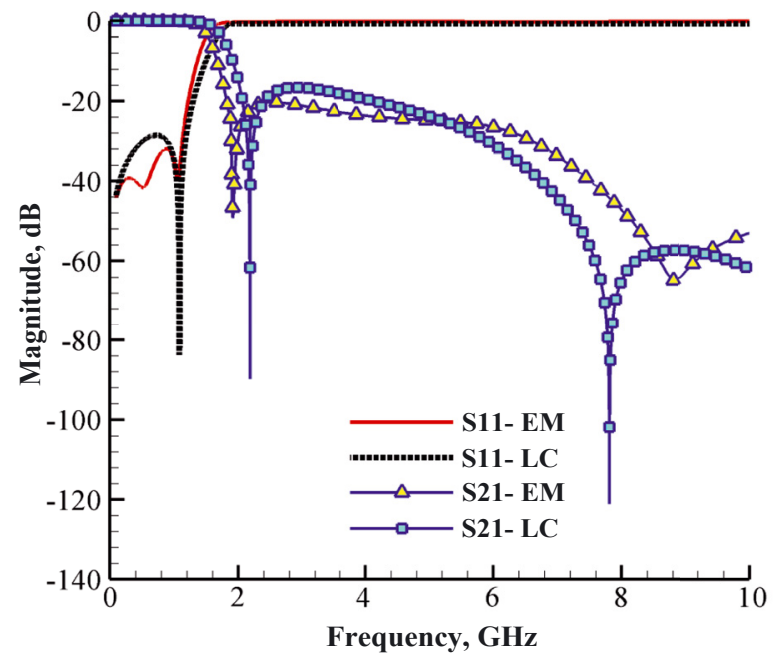

(c)

Figure 4: Proposed LPF (a) LC equal structure, (b) layout structure, (c) simulation results up to $10 \mathrm{GHz}$.

To increase the level of suppression in " $a, b$ and $c$ " points, two attenuator cells are located in series with the prototype LPF. Eventually, the final LPF with $-3 \mathrm{~dB}$ cut-off frequency at $1.50 \mathrm{GHz}$ is designed. The total equivalent $\mathrm{LC}$ circuit is shown in Figure 4(a). As seen in Figure 4(b), the complementary dimensions of the proposed LPF are (All in millimeters): $L_{9}=1.34, L_{10}=4.5, L_{11}=0.2, L_{12}=7.35, W_{8}=1$. Also, the LPF LC equal values are (Units: $C_{t i}, \mathrm{pF} ; L_{t i}, \mathrm{nH}, i=1,2,3 \ldots$ );

$L_{t 1}=0.89, L_{t 2}=0.13, L_{t 3}=4.86, L_{t 4}=4.23, L_{t 5}=1.39$, $L_{t 6}=0.52, L_{t 7}=0.08, L_{t 8}=0.39, L_{t 9}=1.12, C_{t 1}=0.2, C_{t 2}=0.19$, $C_{t 3}=0.23, C_{t 4}=0.11, C_{t 5}=0.45, C_{t 6}=0.41, C_{t 7}=0.97$, $C_{t 8}=0.85, C_{t 9}=0.18$ (The values of the inductances and capacitances are calculated using Eqs. (1)-(4)). The results of the EM and LC simulations are shown in Figure 4(b), up to $10 \mathrm{GHz}$.
Table 2: Substrate details.

\begin{tabular}{lrr}
\hline Relative dielectric constant & Loss tangent & Thickness (mils) \\
\hline 2.2 & 0.0009 & 15 \\
\hline
\end{tabular}

\section{Simulation and experimental results}

The proposed LPF is designed and fabricated on an RT/ Duroid 5880 substrate. The substrate details are mentioned in Table 2.

The photograph and $S$-parameters of the fabricated and simulated LPF are shown in Figure 5(a) and (b), respectively. The measured results are obtained by the Agilent Network Analyser N5230A. The proposed LPF's cutoff frequency is $1.50 \mathrm{GHz}$, the stopband bandwidth is expanded from $1.84 \mathrm{up}$ to $21.39 \mathrm{GHz}$ with $20 \mathrm{~dB}$ attenuation level, and this stopband region is about $13 \mathrm{fc}$. In the passband region, a suppression level of $29.7 \mathrm{~dB}$ is obtained. The size of the LPF circuit is only about $22 \times 6.45 \mathrm{~mm}^{2}$. The designed filter has a high Figure of Merit (FOM) equal to

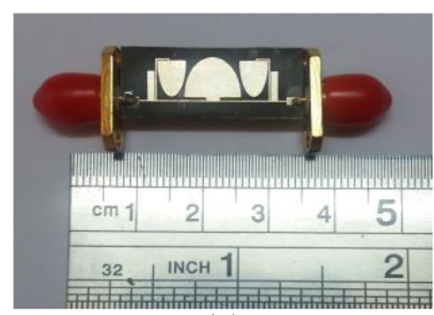

(a)

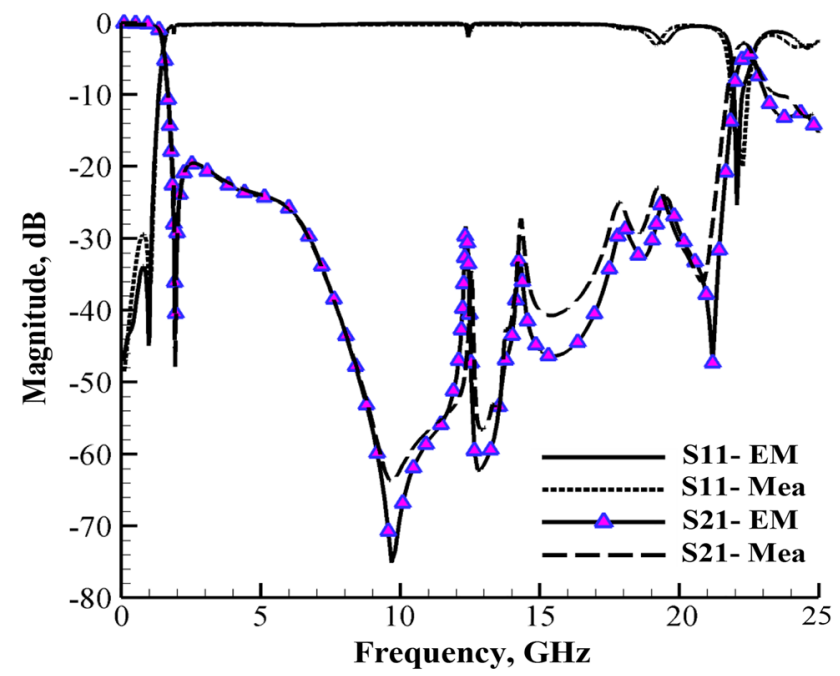

(b)

Figure 5: LPF performance (a) photograph (b) EM and measured simulation up to $25 \mathrm{GHz}$. 
Table 3: Comparison of LPFs performance.

\begin{tabular}{|c|c|c|c|c|c|c|}
\hline Ref. & 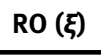 & RSB & SF & $\operatorname{NCS}\left(\lambda_{g}{ }^{2}\right)$ & $\mathbf{A F}$ & FOM \\
\hline 3] & 48.5 & 1.49 & 2.5 & $0.160 \times 0.080$ & 1 & 14,062 \\
\hline [4] & 36 & 0.96 & 1.2 & $0.350 \times 0.200$ & 1 & 600 \\
\hline [6] & 57.8 & 1.61 & 3.5 & $0.120 \times 0.100$ & 1 & 27,142 \\
\hline [7] & 84.7 & 1.51 & 2 & $0.143 \times 0.156$ & 1 & 11,625 \\
\hline [8] & 188.8 & 0.92 & 2 & $0.200 \times 0.180$ & 1 & 9681 \\
\hline [9] & 25.3 & 1.34 & 2 & $0.186 \times 0.101$ & 1 & 3626 \\
\hline [11] & 74 & 1.74 & 2.4 & $0.090 \times 0.136$ & 1 & 25,274 \\
\hline [13] & 100 & 1.58 & 2 & $0.670 \times 0.110$ & 1 & 4288 \\
\hline [14] & 104 & 1.80 & 2.5 & $0.189 \times 0.121$ & 1 & 22,510 \\
\hline [20] & 52.8 & 1.52 & 2 & $0.081 \times 0.113$ & 1 & 17,638 \\
\hline This work & 84 & 1.69 & 2 & $0.151 \times 0.044$ & 1 & 42,759 \\
\hline
\end{tabular}

RO means roll-off rate or selectivity according to 3 and $40 \mathrm{~dB}$ suppressing points [19]. RSB, relative stopband bandwidth; SF, suppression factor; NCS, normalized circuit size; AF, architecture factor; FOM, figure of merit.

42,759. For comparison, Table 3 shows a summary of key LPF performance metrics [12], for current designs.

\section{Conclusions}

In this paper, a simple and compact microstrip LPF with an ultra-wide stopband based on elliptical resonators is designed, fabricated and measured. The presented LPF has a $-3 \mathrm{~dB}$ cut-off frequency at $1.50 \mathrm{GHz}$. The proposed filter has many desirable features, each of which depicts proper characteristics for a LPF theoretically and practically, such as compact size, return loss better than $29 \mathrm{~dB}$, and ultrawide stopband from $1.84 \mathrm{GHz}$ to $21.39 \mathrm{GHz}$. With all these good features, the proposed LPF is recommended for modern communications systems.

Author contributions: All the authors have accepted responsibility for the entire content of this submitted manuscript and approved submission.

Research funding: None declared.

Conflict of interest statement: The authors declare no conflicts of interest regarding this article.

\section{References}

[1] J. S. G. Hong and M. J. Lancaster, Microstrip Filters for RF/ Microwave Applications, New York, John Wiley \& Sons, 2004.

[2] D. M. Pozar, Microwave Engineering, 4th ed. New York, Wiley, 2011.

[3] S. S. Karthikeyan and R. S. Kshetrimayum, "Compact and wide stopband lowpass filter using open complementary split ring resonator and defected ground structure," Radioengineering, vol. 24, no. 3, pp. 708-711, 2015.

[4] S. Sen, T. Moyra, and D. Sarkar, "Modelling and validation of microwave LPF using modified rectangular split ring resonators (SRR) and defected structures," AEU-Int. J. Electron. Commun., vol. 88, pp. 1-10, 2018.

[5] M. Mirzaee and B. S. Virdee, "Realization of highly compact planar lowpass filter for UWB RFID applications," Electron. Lett., vol. 49, no. 22, pp. 1396-1398, 2013.

[6] B. Zhang, S. Li, and J. Huang, "Compact lowpass filter with wide stopband using coupled rhombic stubs," Electron. Lett., vol. 51, no. 3, pp. 264-266, 2015.

[7] M. Hayati, M. Gholami, H. S. Vaziri, et al., "Design of microstrip lowpass filter with wide stop-band and sharp roll-off using hexangular shaped resonator," Electron. Lett., vol. 51, no. 1, pp. 69-71, 2015.

[8] M. Xio, G. Sun, and X. Li, “A lowpass filter with compact size and sharp roll-off,” IEEE Microw. Compon. Lett., vol. 25, no. 12, pp. 790-792, 2015.

[9] B. Hiedari and F. Shama, "A harmonics suppressed microstrip cell for integrated applications," AEU-Int. J. Electron. Commun., vol. 83, pp. 519-522, 2018.

[10] S. Jiang and J. Xu, "Compact microstrip lowpass filter with ultrawide stopband based on dual-plane structure," Electron. Lett., vol. 53, no. 9, pp. 607-609, 2017.

[11] C. J. Chen, C. H. Sung, and Y. D. Su, "A multi-stub lowpass filter," IEEE Microw. Wireless Compon. Lett., vol. 25, no. 8, pp. 532-534, 2015.

[12] S. Lotfi and M. Hayati, "Compact lowpass filter with ultra-wide stopband using analysed triangular shaped resonator," Electron. Lett., vol. 53, no. 15, pp. 1050-1052, 2017.

[13] M. Hayati, M. Ekhteraei, and F. Shama, "Compact lowpass filter with flat group delay using lattice-shaped resonator," Electron. Lett., vol. 53, no. 7, pp. 475-476, 2017.

[14] S. Jiang and J. Xu, "Sharp roll-off planar lowpass filter with ultrawide stopband up to $40 \mathrm{GHz}$," Electron. Lett., vol. 53, no. 11, pp. 734-735, 2017.

[15] A. Lalbakhsh, M. B. Jamshidi, H. Siahkamarl, et al., "A compact lowpass filter for satellite communication systems based on transfer function analysis," AEU-Int. J. Electron. Commun., vol. 124, 2020, https://doi.org/10.1016/j.aeue.2020.153318.

[16] Q. Yang, Y. C. Jiao, and Z. Zhang, "Dual-wideband BPF with wide upper stopband using shorted stepped-impedance stub-loaded lowpass filter," Electron. Lett., vol. 52, no. 19, pp. 1615-1616, 2016.

[17] Q. Li, Y. Zhang, and Y. Fan, “Compact ultra-wide stopband lowpass filter using multimode resonators," Electron. Lett., vol. 51, no. 14, pp. 1084-1085, 2015.

[18] J. D. Ruiz, F. L. M. Viviente, and J. Hinojosa, “Optimization of chirped and tapered microstrip Koch fractal electromagnetic bandgap structures for improved low-pass filter design," IET Microw, Antennas Propag., vol. 9, no. 9, pp. 889-897, 2015.

[19] S. Lotfi, S. V. Makki, and G. R. Karimi, “Compact microstrip lowpass filter with ultra-wide stopband and sharp roll-off based on modified hairpin resonator," Int. J. Eng. Technol., vol. 2, no. 2, pp. 109-121, 2014.

[20] S. Liu, J. Xu, and Z. Xu, "Compact lowpass filter with wide stopband using stepped impedance hairpin units," Electron. Lett., vol. 51, no. 1, pp. 67-69, 2015. 
[21] X. Chen, L. Zhang, et al., "Compact lowpass filter with wide stopband bandwidth," Microw. Opt. Technol. Lett., vol. 57, no. 2, pp. 367-371, 2015.

[22] M. B. Jamshidl, A. Lalbakhsh, B. Mohamadzade, et al., "A novel neural-based approach for design of microstrip filters," AEU-Int. J. Electron. Commun., vol. 110, 2019, https://doi.org/10.1016/j. aeue.2019.152847.

[23] G. Karimi, S. Lotfi, and H. Siahkamari, "Design of microstrip lowpass filter with sharp roll-off using elliptical and radial resonators," Frequenz, vol. 71, no. 7-8, pp. 349-356, 2017.

[24] A. Abdipour, A. Abdipour, and S. Lotfi, "A lowpass filter with sharp roll-off and high relative stopband bandwidth using asymmetric high-low impedance patches," Radioengineering, vol. 24, no. 3, pp. 712-716, 2015.

[25] M. Hayati, S. Zarghami, and F. Shama, "Compact microstrip lowpass filter with very sharp roll-off using meandered line resonators," Wireless Network., vol. 1, no. 1, 2020, https://doi. org/10.1007/s11276-020-02509-7.

[26] M. Kazemi, S. Lotfi, H. Siahkamari, et al., "UWB bandpass filter with ultra-wide stopband based on ring resonator," Frequenz, vol. 72, no. 5-6, pp. 245-252, 2018.
[27] S. Lotfi, S. Roshani, S. Roshani, et al., "Wilkinson power divider with band-pass filtering response and harmonics suppression using open and short stubs," Frequenz, vol. 74, no. 5-6, pp. 169-176, 2020.

[28] M. Jamshidi, A. Lalbakhsh, S. Lotfi, et al., "A neuro-based approach to designing a Wilkinson power divider," Int. J. RF Microw. Computer-Aided Eng., vol. 30, no. 3, 2020, https://doi. org/10.1002/mmce.22091.

[29] S. Lotfi, S. Roshani, and S. Roshani, “Design of a miniaturized planar microstrip Wilkinson power divider with harmonic cancellation," Turk. J. Electr. Eng. Comput. Sci., vol. 28, no. 6, pp. 3126-3136, 2020.

[30] M. Kumar, G. Sen, S. K. Islam, et al., “Miniaturization and harmonic suppression of power divider using coupled line section for high power applications," Radioengineering, vol. 29 , no. 2, 2020, https://doi.org/10.13164/re.2020. 0336.

[31] M. B. Jamshidi, S. Roshani, J. Talla, et al., "Size reduction and performance improvement of a microstrip Wilkinson power divider using a hybrid design technique," Sci. Rep., vol. 11, no. 1, pp. 1-5, 2021. 